\title{
The probability distribution of the ambiguity bootstrapped GNSS baseline
}

\author{
P. J. G. Teunissen \\ Department of Mathematical Geodesy and Positioning, Delft University of Technology, Thijsseweg 11, \\ 2629 JA Delft, The Netherlands \\ e-mail: p.j.g.teunissen@geo.tudelft.nl; Tel.: + +31-15-278-2558; Fax: + +31-15-278-3711
}

Received: 28 September 2000 / Accepted: 11 January 2001

\begin{abstract}
The purpose of carrier phase ambiguity resolution is to improve upon the quality of the estimated global navigation satellite system baseline by means of the integer ambiguity constraints. However, in order to evaluate the quality of the ambiguity resolved baseline rigorously, its probability distribution is required. This baseline distribution depends on the random characteristics of the estimated integer ambiguities, which in turn depend on the chosen integer estimator. In this contribution is presented an exact and closed-form expression for the baseline distribution in the case that use is made of integer bootstrapping. Also presented are the bootstrapped probability mass function and easy-to-compute measures for the bootstrapped baseline's probability of concentration.
\end{abstract}

Key words: Global Navigation Satellite System Ambiguity Resolution - Integer Bootstrapping Probability Mass Function - Multi-modal Baseline Distribution

\section{Introduction}

Global navigation satellite system (GNSS) ambiguity resolution is the process of resolving the unknown cycle ambiguities of double-difference (DD) carrier phase data as integers. Ambiguity resolution applies to a great variety of GNSS models which are currently in use in navigation, surveying, geodesy and geophysics. An overview of these models, together with their applications, can be found in textbooks such as those of Leick (1995), Parkinson and Spilker (1996), Hofmann-Wellenhof et al. (1997), Strang and Borre (1997) and Teunissen and Kleusberg (1998).

The purpose of ambiguity resolution is to improve significantly upon the estimated baseline parameters by means of including the integer ambiguity constraints. When evaluating the quality of the GNSS baseline, however, we usually rely on the assumption that the integer ambiguities are deterministic. Strictly speaking this is not correct, as was pointed out in Teunissen (1990). The integer ambiguities are estimated from the data and since the data are modelled as random variates, the estimated ambiguities are random variates too. They have their own probability distribution, despite their integerness. For a proper evaluation of the quality of the GNSS baseline, we should therefore take the random characteristics of the estimated integer ambiguities into account as well. It is the purpose of this contribution to show how the quality of the GNSS baseline should be evaluated in the case that the integer ambiguities are estimated by means of the bootstrapping principle.

This contribution is organized as follows. In Sect. 2 we give a brief review of the principles involved in ambiguity resolution. We describe the class of admissible integer estimators and show how their probability mass functions, which depend on the ambiguity pull-in regions, contribute to the probability distribution of the ambiguity resolved baseline. In Sect. 3 we introduce the bootstrapped ambiguity estimator and show how this integer estimator is related to sequential conditional least-squares (LS) and to the unique triangular decomposition of the ambiguity variance-covariance matrix. The link with the triangular decomposition is used to describe the bootstrapped pull-in regions and to show that the bootstrapped estimator is an admissible integer estimator. A probabilistic description of integer bootstrapping is given in Sect. 4. Exact and closed-form expressions are derived for the bootstrapped probability mass function and for the distribution of the ambiguity bootstrapped baseline. These results enable us to study and evaluate for the first time the probabilistic properties of the bootstrapped baseline rigorously. We also present easy-to-compute measures for the bootstrapped baseline's probability of concentration. 


\section{GNSS ambiguity resolution}

\subsection{The 'fixed' baseline}

Any GNSS model can be cast in the following system of linear(ized) observation equations:

$y=A a+B b+e$

where $y$ is the given GNSS data vector of order $m, a$ and $b$ are the unknown parameter vectors respectively of order $n$ and $p$, and $e$ is the noise vector. The data vector $y$ will usually consist of the 'observed minus computed' single-, dual- or triple-frequency DD phase and/or pseudorange (code) observations accumulated over all observation epochs. The entries of vector $a$ are then the DD carrier phase ambiguities, expressed in units of cycles rather than range. They are known to be integers, $a \in Z^{n}$. The entries of the vector $b$ will consist of the remaining unknown parameters, such as for instance baseline components (coordinates) and possibly atmospheric delay parameters (troposphere, ionosphere). They are known to be real valued, $b \in R^{p}$. Although vector $b$ may contain other or more real-valued unknown parameters than only those of the baseline(s), we will in this contribution, as a matter of terminology, still call its estimator the baseline estimator.

The procedure which is usually followed for solving the GNSS model of Eq. (1) can be divided into three steps (Teunissen 1993). In the first step we simply discard the integer constraints $a \in Z^{n}$ on the ambiguities and perform a standard adjustment. As a result we obtain the (real-valued) estimates of $a$ and $b$, together with their variance-covariance matrix

$$
\left[\begin{array}{l}
\hat{a} \\
\hat{b}
\end{array}\right],\left[\begin{array}{cc}
Q_{\hat{a}} & Q_{\hat{a} \hat{b}} \\
Q_{\hat{b} \hat{a}} & Q_{\hat{b}}
\end{array}\right]
$$

This solution is referred to as the 'float' solution. In the second step the 'float' ambiguity estimate $\hat{a}$ is used to compute the corresponding integer ambiguity estimate

$\check{a}_{S}=S(\hat{a})$

with $S: R^{n} \mapsto Z^{n}$ a mapping from the $n$-dimensional space of real numbers to the $n$-dimensional space of integers. Once the integer ambiguities are computed, they are used in the third and final step to correct the 'float' estimate of $b$. As a result we obtain the ambiguity resolved baseline solution

$\check{b}_{S}=\hat{b}-Q_{\hat{b} \hat{a}} Q_{\hat{a}}^{-1}\left(\hat{a}-\check{a}_{S}\right)$

This solution is usually referred to as the 'fixed' baseline. The quality of the estimator $\breve{b}_{S}$ depends on the quality of the 'float' solution, $\hat{a}$ and $\hat{b}$, and on the quality of the integer estimator $\check{a}_{S}$. Thus in order to determine the probability density function (PDF) of the 'fixed' baseline, we need in addition to the PDFs of the 'float' solution also the probability distribution of the integer estimator $\check{a}_{S}$. This distribution, however, depends on the type of integer estimator chosen. Different choices of the map $S: R^{n} \mapsto Z^{n}$ will result in different integer estimators and will thus also produce differences in the probability distribution.

\subsection{Admissible ambiguity estimators}

There exists a whole class of integer estimators from which we can choose. In order to introduce this class, we start from the map $S: R^{n} \mapsto Z^{n}$. Due to the discrete nature of $Z^{n}$, the map $S$ will not be one-to-one, but instead a many-to-one map. This implies that different real-valued ambiguity vectors will be mapped to the same integer vector. We can therefore assign a subset $S_{z} \subset R^{n}$ to each integer vector $z \in Z^{n}$

$S_{z}=\left\{x \in R^{n} \mid z=S(x)\right\}, \quad z \in Z^{n}$

The subset $S_{z}$ contains all real-valued ambiguity vectors that will be mapped by $S$ to the same integer vector $z \in Z^{n}$. This subset is referred to as the pull-in region of $z$ (Jonkman 1998; Teunissen 1998). It is the region from which all ambiguity 'float' solutions are pulled to the same 'fixed' ambiguity vector $z$. Thus

$\check{a}_{S}=z \Longleftrightarrow \hat{a} \in S_{z}$

Since the pull-in regions define the integer estimator completely, we can define classes of integer estimators by imposing various conditions on the pull-in regions. The class of admissible integer ambiguity estimators is defined as follows.

Definition (admissible integer estimators). The integer estimator $\check{a}_{S}=S(\hat{a})$ is said to be admissible when its pullin regions $S_{z}=\left\{x \in R^{n} \mid z=S(x)\right\}, z \in Z^{n}$, satisfy

(i) $\cup_{z \in Z^{n}} S_{z}=R^{n}$

(ii) $\operatorname{Int} S_{z_{1}} \cap \operatorname{Int} S_{z_{2}}=\emptyset, \forall z_{1}, z_{2} \in Z^{n}, z_{1} \neq z_{2}$

(iii) $S_{z}=z+S_{0}, \forall z \in Z^{n}$

This definition is motivated as follows. Each one of the above three conditions describes a property which it seems reasonable is possessed by an arbitrary integer ambiguity estimator. The first condition states that the pull-in regions should not leave any gaps and the second that they should not overlap. The absence of gaps is needed in order to be able to map any 'float' solution $\hat{a} \in R^{n}$ to $Z^{n}$, while the absence of overlaps is needed to guarantee that the 'float' solution is mapped to just one integer vector. Note that we allow the pull-in regions to have common boundaries. This is permitted if we assume to have zero probability that $\hat{a}$ lies on one of the boundaries. This will be the case when the PDF of $\hat{a}$ is continuous.

The third and last condition follows from the requirement that $S(x+z)=S(x)+z, \forall x \in R^{n}, z \in Z^{n}$. This condition is also a reasonable one. It states that when the 'float' solution is perturbed by $z \in Z^{n}$, the corresponding integer solution is perturbed by the same amount. This property allows us to apply the integer remove-restore technique: $S(\hat{a}-z)+z=S(\hat{a})$. It therefore allows us to work with the fractional parts of the entries of $\hat{a}$, instead of with its complete entries. 
The simplest example of an admissible estimator is the one that corresponds to a componentwise integer rounding of the 'float' ambiguity vector $\hat{a}=\left(\hat{a}_{1}, \ldots, \hat{a}_{n}\right)^{T}$. Its corresponding pull-in regions are given as

$S_{R, z}=\left\{x \in R^{n}|| x_{i}-z_{i} \mid \leq \frac{1}{2}, i=1, \ldots, n\right\}$

They are the multivariate versions of the integercentered unit square. The above admissibility conditions are satisfied, since all the unit squares are translated copies of one another and together they fill the whole space without gaps and overlaps.

\subsection{The PMF of admissible estimators}

The distribution of an admissible ambiguity estimator can be determined once its pull-in regions are known and once the PDF of the 'float' solution is given. Since the integer estimator $\check{a}_{S}$ is by definition of the discrete type, its distribution will be a probability mass function (PMF). It has zero masses at non-integer points and non-zero masses at some or all integer points. The PMF of $\check{a}_{S}$ will be denoted as $P\left(\check{a}_{S}=z\right)$, with $z \in Z^{n}$. The PDF of the 'float' ambiguity solution $\hat{a}$ will be denoted as $p_{\hat{a}}(x)$.

The PMF of $\check{a}_{S}$ can now be obtained as follows. Since the integer estimator is defined as $\check{a}_{S}=z \Longleftrightarrow \hat{a} \in S_{z}$, it follows that $P\left(\check{a}_{S}=z\right)=P\left(\hat{a} \in S_{z}\right)$. The PMF of $\check{a}_{S}$ therefore follows as

$P\left(\check{a}_{S}=z\right)=\int_{S_{z}} p_{\hat{a}}(x) \mathrm{d} x, \quad z \in Z^{n}$

The probability that $\check{a}_{S}$ coincides with $z$ is given by the integral of the PDF $p_{\hat{a}}(x)$ over the pull-in region $S_{z} \subset R^{n}$.

Note that the above expression holds for any distribution the 'float' ambiguities $\hat{a}$ might have. In most GNSS applications, however, we usually assume the vector of observables $y$ to be normally distributed. In that case the 'float' solutions $\hat{a}$ and $\hat{b}$, both being linear estimators, will be normally distributed too. In the remainder of this contribution we will therefore assume that the 'float' solutions are normally distributed as

$$
\left[\begin{array}{l}
\hat{a} \\
\hat{b}
\end{array}\right] \sim \mathrm{N}\left(\left[\begin{array}{l}
a \\
b
\end{array}\right],\left[\begin{array}{cc}
Q_{\hat{a}} & Q_{\hat{a} \hat{b}} \\
Q_{\hat{b} \hat{a}} & Q_{\hat{b}}
\end{array}\right]\right)
$$

\subsection{The PDF of the 'fixed' baseline}

In order to determine the PDF of the 'fixed' baseline estimator $\breve{b}_{S}$, we need to propagate the uncertainty of the 'float' solution, $\hat{a}$ and $\hat{b}$, as well as the uncertainty of the integer solution $\check{a}_{S}$ through Eq. (4). Should we neglect the random character of the integer solution and therefore consider the ambiguity vector $\check{a}_{S}$ as deterministic and equal to, say, $z$, then the PDF of $\breve{b}_{S}$ would equal the conditional baseline distribution $p_{\hat{b} \mid \hat{a}}(x \mid y=z)=\frac{1}{\sqrt{\operatorname{det} Q_{\hat{b} \mid \hat{a}}}(2 \pi)^{\frac{1}{2} p}} \exp \left\{-\frac{1}{2}\left\|x-b_{\mid \hat{a}=z}\right\|_{Q_{\hat{b} \mid \hat{a}}}^{2}\right\}$

with conditional mean $b_{\mid \hat{a}=z}=b-Q_{\hat{b} \hat{a}} Q_{\hat{a}}^{-1}(a-z)$, conditional variance matrix $Q_{\hat{b} \mid \hat{a}}=Q_{\hat{b}}-Q_{\hat{b} \hat{a}} Q_{\hat{a}}^{-1} Q_{\hat{a} \hat{b}}$ and $\|\cdot\|_{Q_{\hat{b} \mid \hat{a}}}^{2}=(\cdot)^{T} Q_{\hat{b} \mid \hat{a}}^{-1}(\cdot)$. However, since $\check{a}_{S}$ is random and not deterministic, the conditional baseline distribution will give a too optimistic description of the quality of the 'fixed' baseline. In order to obtain a correct description of the 'fixed' baseline's PDF, the integer ambiguity's PMF needs to be considered. As the following theorem shows, this results in a baseline distribution, which generally will be multi-modal.

Theorem 1 (distribution of the 'fixed' baseline). Let the 'float' solution, $\hat{a}$ and $\hat{b}$, be distributed as in Eq. (9), let $\check{a}_{S}$ be an admissible integer ambiguity estimator and let the 'fixed' baseline $\breve{b}_{S}$ be given as in Eq. (4). The PDF of $\breve{b}_{S}$ then reads

$p_{\check{b}_{S}}(x)=\sum_{z \in Z^{n}} p_{\hat{b} \mid \hat{a}}(x \mid y=z) P\left(\check{a}_{S}=z\right)$

This theorem was first introduced and proved in Teunissen (1999). Note that the theorem is quite general, since the result of Eq. (11) holds true for any admissible integer estimator. Also note that, although the model of Eq. (1) is linear and the observables normally distributed, the distribution of the 'fixed' baseline is not normal. As the theorem shows, the 'fixed' baseline distribution equals an infinite sum of weighted conditional baseline distributions. These conditional baseline distributions $p_{\hat{b} \mid \hat{a}}(x \mid y=z)$ are shifted versions of one another. The size and direction of the shift is governed by $Q_{\hat{b} \hat{a}} Q_{\hat{a}}^{-1} z, z \in Z^{n}$. Each of the conditional baseline distributions in the infinite sum is downweighted. These weights are given by the probability masses of the distribution of the integer ambiguity estimator $\check{a}_{S}$. This shows that the dependence of the 'fixed' baseline distribution on the choice of integer estimator is only felt through the weights $P\left(\check{a}_{S}=z\right)$.

In order to describe the quality of the 'fixed' baseline, we would like to know how close we can expect the baseline estimate $\check{b}_{S}$ to be to the unknown but true baseline value $b$. We therefore would like to be able to evaluate the peakedness of the PDF $p_{\breve{b}_{S}}(x)$. This implies, in probabilistic terms, that we would like to be able to evaluate the probability that $\check{b}_{S} \in R$, with $R$ a subset of $R^{p}$, centered at $b$. This probability is given by

$P\left(\check{b}_{S} \in R\right)=\int_{R} p_{\breve{b}_{S}}(x) \mathrm{d} x$

However, in order to evaluate this probability, we also need, as Eq. (11) shows, an explicit expression for the PMF of $\check{a}_{S}$. This PMF depends on the integer estimator chosen. In the remainder of this contribution we will consider one of the more popular integer estimators, namely the bootstrapped ambiguity estimator. This estimator and its properties will be introduced in the next section. 


\section{Integer bootstrapping}

\subsection{Sequential conditional LS}

To prepare for our discussion of the bootstrapped estimator, we first consider the adjustment principles of 'conditional LS' and 'sequential conditional LS'. These principles form the basis of the bootstrapped estimator. We will show how the sequential conditional LS ambiguity estimator is constructed and how it is related to the unique lower triangular decomposition of the ambiguity variance-covariance matrix. We commence with the principle of conditional LS estimation. The following corollary is a well-known result from standard adjustment theory.

Corollary 1 (conditional $L S$ ). Let the expectation and dispersion of $\hat{a}_{I}=\left(\hat{a}_{1}, \ldots, \hat{a}_{i-1}\right)^{T} \in R^{i-1}$ and $\hat{a}_{i} \in R$ be given as

$E\left\{\left[\begin{array}{l}\hat{a}_{I} \\ \hat{a}_{i}\end{array}\right]\right\}=\left[\begin{array}{l}a_{I} \\ a_{i}\end{array}\right], \quad D\left\{\left[\begin{array}{l}\hat{a}_{I} \\ \hat{a}_{i}\end{array}\right]\right\}=\left[\begin{array}{cc}Q_{I} & Q_{I i} \\ Q_{i I} & \sigma_{i}^{2}\end{array}\right]$

Then the LS estimator of $a_{i}$, when $a_{I}$ is constrained to the fixed vector $z_{I}$, is given as

$\hat{a}_{i \mid I}=\hat{a}_{i}-Q_{i I} Q_{I}^{-1}\left(\hat{a}_{I}-z_{I}\right)$

The estimator $\hat{a}_{i \mid I}$ is referred to as the conditional LS ambiguity estimator. It is conditioned on fixing the previous ambiguities to the values $z_{j}, j=1, \ldots,(i-1)$. Note that $\hat{a}_{i \mid I}$ and $\hat{a}_{I}$ are uncorrelated. This is an important property that will be used repeatedly in the following.

The above result can be used to derive a sequential version of the conditional LS estimator. For $i=2$, we obtain the scalar version of Eq. (13)

$\hat{a}_{2 \mid 1}=\hat{a}_{2}-\sigma_{21} \sigma_{1}^{-2}\left(\hat{a}_{1}-z_{1}\right)$

in which $\hat{a}_{2 \mid 1}$ is uncorrelated with $\hat{a}_{1}$. For $i=3$, the conditional LS estimator $\hat{a}_{3 \mid 2,1}$ follows from fixing the two ambiguities $a_{1}$ and $a_{2}$ to the values $z_{1}$ and $z_{2}$. Note, however, that since $\hat{a}_{3 \mid 2,1}$ is invariant to any regular transformation of $\hat{a}_{1}, \hat{a}_{2}$, we may as well fix $\hat{a}_{1}$ and $\hat{a}_{2 \mid 1}$ to the values $z_{1}$ and $z_{2}$. This has the advantage that matrix $Q_{I}$ of Eq. (13) becomes diagonal. As a result we obtain

$\hat{a}_{3 \mid 2,1}=\hat{a}_{3}-\sigma_{3,1} \sigma_{1}^{-2}\left(\hat{a}_{1}-z_{1}\right)-\sigma_{3,2 \mid 1} \sigma_{2 \mid 1}^{-2}\left(\hat{a}_{2 \mid 1}-z_{2}\right)$

in which $\hat{a}_{3 \mid 2,1}$ is uncorrelated with both $\hat{a}_{1}$ and $\hat{a}_{2 \mid 1}$. It will be clear that we may continue in this way to obtain the corresponding expressions for the next and following ambiguities as well. The result is summarized in the following corollary.

Corollary 2 (sequential conditional $L S$ ). The conditional LS estimator $\hat{a}_{i \mid I}$ can be computed sequentially as

$\hat{a}_{i \mid I}=\hat{a}_{i}-\sum_{j=1}^{i-1} \sigma_{i, j \mid J} \sigma_{j \mid J}^{-2}\left(\hat{a}_{j \mid J}-z_{j}\right), i=1, \ldots, n$

where $\sigma_{i, j \mid J}$ denotes the covariance between $\hat{a}_{i}$ and $\hat{a}_{j \mid J}$, and $\sigma_{j \mid J}^{2}$ is the variance of $\hat{a}_{j \mid J}$. For $i=1, \hat{a}_{i \mid I}$ is set equal to $\hat{a}_{1}$.
With this result we are now also in a position to show how sequential conditional LS estimation relates to the unique lower triangular decomposition of the ambiguity variance-covariance matrix. From Eq. (16) it follows that the difference $\left(\hat{a}_{i}-z_{i}\right)$ may be written in terms of the differences $\left(\hat{a}_{j \mid J}-z_{j}\right), j=1, \ldots, i$, as

$\left(\hat{a}_{i}-z_{i}\right)=\left(\hat{a}_{i \mid I}-z_{i}\right)+\sum_{j=1}^{i-1} \sigma_{i, j \mid J} \sigma_{j \mid J}^{-2}\left(\hat{a}_{j \mid J}-z_{j}\right)$

When written out in vector-matrix form, this gives

$$
\left[\begin{array}{c}
\hat{a}_{1}-z_{1} \\
\hat{a}_{2}-z_{2} \\
\vdots \\
\hat{a}_{n}-z_{n}
\end{array}\right]=\left[\begin{array}{cccc}
1 & & & \\
l_{21} & 1 & & \\
\vdots & \vdots & \ddots & \\
l_{n 1} & l_{n 2} & \ldots & 1
\end{array}\right]\left[\begin{array}{c}
\hat{a}_{1}-z_{1} \\
\hat{a}_{2 \mid 1}-z_{2} \\
\vdots \\
\hat{a}_{n \mid N}-z_{n}
\end{array}\right]
$$

with $l_{i j}=\sigma_{i, j \mid J} \sigma_{j \mid J}^{-2}$, for $1 \leq j<i \leq n$. Since the sequential conditional LS ambiguities are mutually uncorrelated, their variance-covariance matrix is diagonal, as a consequence of which the variance-covariance matrix of the $\hat{a}_{i}$ is given a triangular decomposition, when applying the error propagation law to Eq. (18). The relation between sequential conditional LS and the triangular decomposition is summarized in the following corollary.

Corollary 3 (the statistics of the triangular decomposition). Let the $\hat{a}_{i}, i=1, \ldots, n$, be collected in the vector $\hat{a}=\left(\hat{a}_{1}, \ldots, \hat{a}_{n}\right)^{T}$ and let their sequential conditional LS estimators be collected in the vector $\hat{a}_{c}=\left(\hat{a}_{1}, \ldots, \hat{a}_{n \mid N}\right)^{T}$. Then $\hat{a}$ and $\hat{a}_{c}$, and their variancecovariance matrices, are related as

$\hat{a}-z=L\left(\hat{a}_{c}-z\right) \quad$ and $\quad Q_{\hat{a}}=L D L^{T}$

where the matrix entries are given as

$(L)_{i j}= \begin{cases}0 & \text { for } 1 \leq i<j \leq n \\ 1 & \text { for } i=j \\ \sigma_{i, j \mid J} \sigma_{j \mid J}^{-2} & \text { for } 1 \leq j<i \leq n\end{cases}$

and $D=\operatorname{diag}\left(\ldots, \sigma_{j \mid J}^{2}, \ldots\right)$.

This triangular decomposition enables a compact description of the bootstrapped pull-in region, as well as preparing us for the derivation of the bootstrapped distribution.

\subsection{The bootstrapped estimator}

We are now in a position to describe the integer bootstrapping principle. In order to compute the sequential conditional LS solutions, we need to specify the $z_{j}$ on which the conditioning takes place. In the case of bootstrapping, $z_{j}$, for $j=1, \ldots, n$, is chosen as the nearest integer of $\hat{a}_{j \mid J}$. Hence, for $\hat{a}_{i \mid I}$ the conditioning takes place on the nearest integers of all previous $i-1$ conditional estimates. The $i$ th component of the bootstrapped solution itself is then given as the nearest integer of $\hat{a}_{i \mid I}$. We thus have the following definition. 
Definition (integer bootstrapping). Let $\hat{a}=\left(\hat{a}_{1}, \ldots, \hat{a}_{n}\right)^{T}$ $\in R^{n}$ be the ambiguity 'float' solution and let $\check{a}_{B}=\left(\check{a}_{B, 1}, \ldots, \check{a}_{B, n}\right)^{T} \in Z^{n}$ denote the corresponding integer bootstrapped solution. The entries of the bootstrapped ambiguity estimator are then defined as

$$
\begin{aligned}
\check{a}_{B, 1} & =\left[\hat{a}_{1}\right] \\
\check{a}_{B, 2} & =\left[\hat{a}_{2 \mid 1}\right]=\left[\hat{a}_{2}-\sigma_{21} \sigma_{1}^{-2}\left(\hat{a}_{1}-\check{a}_{B, 1}\right)\right] \\
& \vdots \\
\check{a}_{B, n} & =\left[\hat{a}_{n \mid N}\right]=\left[\hat{a}_{n}-\sum_{j=1}^{n-1} \sigma_{n, j \mid J} \sigma_{j \mid J}^{-2}\left(\hat{a}_{j \mid J}-\check{a}_{B, j}\right)\right]
\end{aligned}
$$

where [.] denotes the operation of rounding to the nearest integer.

As the definition shows, the bootstrapped estimator can be seen as a generalization of the method of 'integer rounding'. If $n$ ambiguities are available, we start with the first ambiguity $\hat{a}_{1}$ and round its value to the nearest integer. Having obtained the integer value of this first ambiguity, the real-valued estimates of all remaining ambiguities are then corrected by virtue of their correlation with the first ambiguity. Then the second, but now corrected, real-valued ambiguity estimate is rounded to its nearest integer. Having obtained the integer value of the second ambiguity, the real-valued estimates of all remaining $n-2$ ambiguities are then again corrected, but now by virtue of their correlation with the second ambiguity. This process is continued until all ambiguities are accommodated. Thus the bootstrapped estimator reduces to 'integer rounding' in the case that correlations are absent, i.e. in case that the ambiguity variance-covariance matrix is diagonal.

Note that the bootstrapped estimator is not unique. Changing the order in which the ambiguities appear in vector $\hat{a}$ will already produce a different bootstrapped estimator. Although the principle of bootstrapping remains the same, every choice of ambiguity parametrization has its own bootstrapped estimator. We will come back to this dependence on the chosen ambiguity parametrization in Sect. 4.2.

\subsection{Bootstrapping is admissible}

We will now show that the bootstrapped estimator belongs to the class of admissible integer estimators. In order to do so, we first need to construct the bootstrapped pull-in regions. They are given in the following corollary.

Corollary 4 (bootstrapped pull-in regions). The pull-in regions of the bootstrapped ambiguity estimator $\check{a}_{B}=\left(\check{a}_{B, 1}, \ldots, \check{a}_{B, n}\right)^{T} \in Z^{n}$ are given as

$$
\begin{gathered}
S_{B, z}=\left\{x \in R^{n}|| c_{i}^{T} L^{-1}(x-z) \mid \leq \frac{1}{2}, i=1, \ldots, n\right\}, \\
\forall z \in Z^{n}
\end{gathered}
$$

where $L$ denotes the unique unit lower triangular matrix of the ambiguity variance-covariance matrix decompo- sition $Q_{\hat{a}}=L D L^{T}$ and $c_{i}$ denotes the $i$ th canonical unit vector having a 1 as its $i$ th entry and zeros otherwise.

Proof. To prove this corollary we make use of the definition of the bootstrapped estimator and of the vector-matrix form of the sequential conditional LS estimator. From Eq. (19) it follows that $\hat{a}_{c}-z=L^{-1}(\hat{a}-z)$. According to Eq. (20), the integer vector $z$ equals the bootstrapped solution when rounding to the nearest integer of each of the components of $\hat{a}_{c}-z$ gives zero, or similarly, when the absolute values of these components are all less than or equal to $1 / 2$. This is equivalent to stating that the absolute values of all the components of $L^{-1}(\hat{a}-z)$ are required to be less than or equal to $1 / 2$, from which the above result follows.

We will now show that the bootstrapped estimator is indeed admissible. The first two conditions of Eq. (7) are easily verified using the definition of the bootstrapped estimator. Since every real-valued vector $\hat{a}$ will be mapped by the bootstrapped estimator to an integer vector, the pull-in regions $S_{B, z}$ cover $R^{n}$ without any gaps. There is also no overlap between the pull-in regions since - apart from boundary ties - any real-valued vector $\hat{a}$ is mapped to not more than one integer vector. To verify the last condition of Eq. (7), we make use of Eq. (21). From

$$
\begin{aligned}
S_{B, z} & =\left\{x \in R^{n}|| c_{i}^{T} L^{-1}(x-z) \mid \leq \frac{1}{2}, i=1, \ldots, n\right\} \\
& =\left\{x \in R^{n}|| c_{i}^{T} L^{-1} y \mid \leq \frac{1}{2}, x=y+z, i=1, \ldots, n\right\} \\
& =S_{B, 0}+z
\end{aligned}
$$

it follows that all bootstrapped pull-in regions are translated copies of $S_{B, 0}$. All pull-in regions therefore have the same shape and the same volume. Their volumes all equal 1 . This can be shown by transforming $S_{B, 0}$ to the unit cube centered at the origin. Consider the linear transformation $y=L^{-1} x$. Then

$$
L^{-1}\left(S_{B, 0}\right)=\left\{y \in R^{n}|| c_{i}^{T} y \mid \leq \frac{1}{2}, i=1, \ldots, n\right\}
$$

equals the unit cube centered at the origin. Since the determinant of the unit lower triangular matrix $L^{-1}$ equals one, and since the volume of the unit cube equals one, it follows that the volume of $S_{B, 0}$ must equal one as well. In order to infer the shape of the bootstrapped pull-in region, we consider the two-dimensional (2-D) case first. Let the lower triangular matrix $L$ be given as

$L=\left[\begin{array}{ll}1 & 0 \\ l & 1\end{array}\right]$

Then

$$
\begin{aligned}
S_{B, 0} & =\left\{x \in R^{2}|| c_{i}^{T} L^{-1} x \mid \leq \frac{1}{2}, i=1,2\right\} \\
& =\left\{x \in R^{2}|| x_{1}\left|\leq \frac{1}{2},\right| x_{2}-l x_{1} \mid \leq \frac{1}{2}\right\}
\end{aligned}
$$

which shows that the 2-D pull-in region equals a parallellogram. Its region is bounded by the two vertical lines $x_{1}=1 / 2$ and $x_{1}=-1 / 2$, and the two parallel slopes $x_{2}=l x_{1}+1 / 2$ and $x_{2}=l x_{1}-1 / 2$. The 
direction of the slope is governed by $l=\sigma_{21} \sigma_{1}^{-2}$. Hence, in the absence of correlation between the two ambiguities, the parallellogram reduces to the unit square. In higher dimensions the above construction of the pull-in region can be continued. In three dimensions, for instance, the intersection of the pull-in region with the $x_{1} x_{2}$-plane remains a parallellogram, while along the third axis the pull-in region becomes bounded by two parallel planes.

\section{The distribution of the bootstrapped baseline}

In this section we present an exact and closed-form expression for the distribution of the ambiguity bootstrapped baseline $\check{b}_{B}$. To this end we first need to derive the probability mass function of the bootstrapped estimator. Once this expression is given, it can be used together with theorem 1 to evaluate the quality of the ambiguity bootstrapped baseline.

\subsection{The PMF of the bootstrapped ambiguity estimator}

As starting point we take the defining integral of the bootstrapped PMF. Since the 'float' ambiguities are assumed to be distributed as $\hat{a} \sim N\left(a, Q_{\hat{a}}\right)$, the multivariate integral which defines the bootstrapped PMF is given as

$$
\begin{aligned}
P\left(\check{a}_{B}=z\right)= & \int_{S_{B, z}}(2 \pi)^{-\frac{n}{2}} \sqrt{\operatorname{det} Q_{\hat{a}}^{-1}} \\
& \times \exp \left\{-\frac{1}{2}(x-a)^{T} Q_{\hat{a}}^{-1}(x-a)\right\} \mathrm{d} x
\end{aligned}
$$

in which the bootstrapped pull-in regions are given as

$S_{B, z}=\left\{x \in R^{n}|| c_{i}^{T} L^{-1}(x-z) \mid \leq \frac{1}{2}, i=1, \ldots, n\right\}, z \in Z^{n}$

Since finding an explicit expression for the PMF is simplest in the scalar case, we first assume $n=1$. In this case bootstrapping becomes equivalent to integer rounding, as a result of which the pull-in regions take the simple form $S_{B, z}=\left\{x \in R|| x-z \mid \leq \frac{1}{2}\right\}, \forall z \in Z$. Each pull-in region is thus equal to an integer-centered interval of length one. The univariate bootstrapped PMF then follows from

$P\left(\check{a}_{B}=z\right)=P\left(\hat{a} \in S_{B, z}\right)=\int_{z-\frac{1}{2}}^{z+\frac{1}{2}} p_{\hat{a}}(x) \mathrm{d} x$

as

$$
\begin{aligned}
P\left(\check{a}_{B}=z\right)= & \Phi\left(\frac{1-2(a-z)}{2 \sigma_{\hat{a}}}\right) \\
& +\Phi\left(\frac{1+2(a-z)}{2 \sigma_{\hat{a}}}\right)-1, z \in Z
\end{aligned}
$$

with

$\Phi(x)=\int_{-\infty}^{x} \frac{1}{\sqrt{2 \pi}} \exp \left\{-\frac{1}{2} v^{2}\right\} \mathrm{d} v$

Note that the PMF is symmetric about $a$. This implies that integer rounding is unbiased. Also note that the peakedness of the PMF is solely driven by the ambiguity standard deviation $\sigma_{\hat{a}}$. Hence, as is the case with the normal distribution, the PMF becomes more peaked when $\sigma_{\hat{a}}$ gets smaller.

The following theorem gives the multivariate version of the bootstrapped PMF. As the theorem shows, it equals a product of univariate PMFs which are similar to the one given above.

Theorem 2 (the integer bootstrapped PMF). Let $\hat{a}$ be distributed as $N\left(a, Q_{\hat{a}}\right), a \in Z^{n}$, and let $\check{a}_{B}$ be the corresponding integer bootstrapped estimator. Then

$$
\begin{aligned}
P\left(\check{a}_{B}=z\right)=\prod_{i=1}^{n}[ & \Phi\left(\frac{1-2 l_{i}^{T}(a-z)}{2 \sigma_{\hat{a}_{i \mid I}}}\right) \\
& \left.+\Phi\left(\frac{1+2 l_{i}^{T}(a-z)}{2 \sigma_{\hat{a}_{i \mid}}}\right)-1\right], z \in Z^{n}
\end{aligned}
$$

with $l_{i}$ the $i$ th column vector of the unit upper triangular matrix $L^{-T}$ and $\sigma_{\hat{a}_{i \mid}}^{2}$ the variance of the $i$ th LS ambiguity obtained through a conditioning on the previous $I=\{1, \ldots,(i-1)\}$ ambiguities.

Proof. In order to prove the theorem, use will be made of the following 'transformation of integrals' formula (Fleming 1977)

$$
\int_{A} f(x) \mathrm{d} x=\int_{g^{-1}(A)} f(g(y))|J g(y)| \mathrm{d} y
$$

in which $J g(y)$ denotes the Jacobian of the regular transformation $x=g(y)$. For our application we choose $g$ as $x=L y+z$, with $L$ the unit lower triangular factor of $Q_{\hat{a}}=L D L^{T}$. Then

$$
\begin{aligned}
P\left(\check{a}_{B}=z\right) & \\
= & \int_{g^{-1}\left(S_{B, z}\right)}(2 \pi)^{-\frac{n}{2}} \sqrt{\operatorname{det} D^{-1}} \exp \left\{-\frac{1}{2}\left(y-L^{-1}(a-z)\right)^{T}\right. \\
& \left.\times D^{-1}\left(y-L^{-1}(a-z)\right)\right\} \mathrm{d} y
\end{aligned}
$$

with the transformed pull-in region

$g^{-1}\left(S_{B, z}\right)=\left\{y \in R^{n}|| c_{i}^{T} y \mid \leq \frac{1}{2}, i=1, \ldots, n\right\}$

Recognizing that $D$ is a diagonal matrix which has the sequential conditional variances as its diagonal entries, and that the transformed pull-in region has become an origin-centered cube with all sides equal to 1 , it follows that the above multivariate integral may be written as a product of 1-D integrals 


$$
\begin{aligned}
P\left(\check{a}_{B}=z\right)= & \prod_{i=1}^{n} \int_{\left|y_{i}\right| \leq \frac{1}{2}} \frac{1}{\sigma_{\hat{a}_{i \mid I}} \sqrt{2 \pi}} \\
& \quad \times \exp \left\{-\frac{1}{2}\left(\frac{y_{i}-l_{i}^{T}(a-z)}{\sigma_{\hat{a}_{i \mid I}}}\right)^{2}\right\} \mathrm{d} y_{i} \\
= & \prod_{i=1}^{n} \int_{-\frac{1+2 l_{i}^{T}(a-z)}{2 \sigma_{\hat{a}_{i \mid I}}}}^{\frac{1-2 l_{i}^{T}(a-z)}{2 \sigma_{\hat{a}_{i \mid I}}}} \frac{1}{\sqrt{2 \pi}} \exp \left\{-\frac{1}{2} v^{2}\right\} \mathrm{d} v
\end{aligned}
$$

from which the result follows.

It follows from Eq. (25) that also the multivariate bootstrapped PMF is symmetric about the mean of $\hat{a}$. This implies that the bootstrapped estimator $\check{a}_{B}$ is an unbiased estimator of $a \in Z^{n}$. Since the 'float' solutions, $\hat{a}$ and $\hat{b}$, are unbiased too, it follows from taking the expectation of Eq. (4) that the bootstrapped baseline is also unbiased. Thus $E\left\{\check{b}_{B}\right\}=b$.

Also note that the PMF reaches its maximum at its point of symmetry. Thus $\max _{z} P\left(\check{a}_{B}=z\right)=P(\check{a}=a)$. This is a reassuring result, since it implies that the probability of correct integer bootstrapped estimation is largest of all probability masses. The probability of correct integer estimation is also referred to as the ambiguity success rate.

Finally observe that the shape of the bootstrapped PMF is completely governed by the ambiguity variancecovariance matrix $Q_{\hat{a}}$. The PMF follows once the triangular factor $L$ and the diagonal matrix $D$ of the decomposition $Q_{\hat{a}}=L D L^{T}$ are given.

\subsection{The quality of the bootstrapped baseline}

We are now in the position to evaluate the quality of the bootstrapped baseline $\check{b}_{B}=\hat{b}-Q_{\hat{b} \hat{a}} Q_{\hat{a}}^{-1}\left(\hat{a}-\check{a}_{B}\right)$. As a measure of confidence, we take

$P\left(\check{b}_{B} \in R\right)=\int_{R} p_{\check{b}_{B}}(x) \mathrm{d} x \quad$ with $\quad R \subset R^{p}$

However, in order to evaluate this integral we first need to make a choice about the shape and location of the subset $R$. Since it is common practice in GNSS positioning to use the variance-covariance matrix of the conditional baseline estimator as a measure of precision for the 'fixed' baseline, the variance-covariance matrix $Q_{\hat{b} \mid \hat{a}}$ will be used to define the shape of the confidence region. For its location, we choose the confidence region to be centered at $b$. After all, we would like to know by how much the baseline estimate $\breve{b}_{B}$ can be expected to differ from the true, but unknown, baseline value $b$. That is, we would like Eq. (26) to be a measure of the bootstrapped baseline's probability of concentration about $b$.

With these choices on shape and location, the confidence region $R$ takes the form
$R=\left\{x \in R^{p} \mid(x-b)^{T} Q_{\hat{b} \mid \hat{a}}^{-1}(x-b) \leq \beta^{2}\right\}$

The size of the region can be varied by varying $\beta$.

The following corollary shows how the bootstrapped baseline's probability of concentration Eq. (26), can be evaluated as a weighted sum of probabilities of noncentral Chi-square distributions.

Corollary 5 (the bootstrapped baseline's probability of concentration). Let $\breve{b}_{B}$ be the ambiguity bootstrapped baseline estimator, $R$ be defined as in Eq. (27), and $\chi^{2}\left(p, \lambda_{z}\right)$ denote the non-central Chi-square distribution with $p$ degrees of freedom and noncentrality parameter $\lambda_{z}$. Then

$P\left(\check{b}_{B} \in R\right)=\sum_{z \in Z^{n}} P\left(\chi^{2}\left(p, \lambda_{z}\right) \leq \beta^{2}\right) P\left(\check{a}_{B}=z\right)$

with

$\lambda_{z}=\left\|\nabla \check{b}_{z}\right\|_{Q_{\hat{b} \mid \hat{a}}}^{2} \quad$ and $\quad \nabla \check{b}_{z}=Q_{\hat{b} \hat{a}} Q_{\hat{a}}^{-1}(z-a)$

Proof. From theorem 1 it follows that $P\left(\check{b}_{B} \in R\right)=\sum_{z \in Z^{n}} P\left(\hat{b}_{\mid \hat{a}=z} \in R\right) P\left(\check{a}_{B}=z\right)$. We will now make use of the fact that if a random vector $u$ of dimension $p \times 1$ is distributed as $u \sim N\left(\mu, Q_{u}\right)$, then the quadratic form $u^{T} Q_{u}^{-1} u$ will have a non-central Chisquare distribution with $p$ degrees of freedom and non-centrality parameter $\lambda=\mu^{T} Q_{u}^{-1} \mu$. If we apply this to the conditional baseline, which is distributed as $\hat{b}_{\mid \hat{a}=z} \sim N\left(b+\nabla \check{b}_{z}, Q_{\hat{b} \mid \hat{a}}\right)$, we obtain $P\left(\hat{b}_{\mid \hat{a}=z} \in R\right)=$ $P\left(\chi^{2}\left(p, \lambda_{z}\right) \leq \beta^{2}\right)$, from which the result follows.

This result shows that the probability of the ambiguity bootstrapped baseline lying inside the ellipsoidal region $R$ centered at $b$ equals an infinite sum of probability products. If we consider the two probabilities of these products separately, two effects are observed: first the probabilistic effect of shifting the conditional baseline estimator away from $b$, and secondly the probabilistic effect of the peakedness or non-peakedness of the bootstrapped PMF. The second effect is related to the expected performance of bootstrapped ambiguity resolution, while the first effect has to do with the sensitivity of the baseline to changes in the values of the integer ambiguities. This effect is measured by the noncentrality parameter $\lambda_{z}$. Since the tail of a non-central Chi-square distribution becomes heavier when the noncentrality parameter increases, while the degrees of freedom remain fixed, $P\left(\chi^{2}\left(p, \lambda_{z}\right) \leq \beta^{2}\right)$ gets smaller when $\lambda_{z}$ gets larger.

The two probabilities in the product reach their maximum values when $z=a$. The following corollary shows how these two maxima can be used to lower bound and to upper bound the probability $P\left(\breve{b}_{B} \in R\right)$. Such bounds are of importance for practical purposes, since it is difficult in general to evaluate this probability exactly.

Corollary 6 (lower and upper bounds). Let $\check{b}_{\mathrm{B}}$ be the ambiguity bootstrapped baseline estimator and let $R$ be defined as in Eq. (27). Then 
$P\left(\hat{b}_{\mid \hat{a}=a} \in R\right) P\left(\check{a}_{B}=a\right) \leq P\left(\check{b}_{B} \in R\right) \leq P\left(\hat{b}_{\mid \hat{a}=a} \in R\right)$

with

$P\left(\hat{b}_{\mid \hat{a}=a} \in R\right)=P\left(\chi^{2}(p, 0) \leq \beta^{2}\right)$

$P\left(\check{a}_{B}=a\right)=\prod_{i=1}^{n}\left[2 \Phi\left(\frac{1}{2 \sigma_{\hat{a}_{i \mid}}}\right)-1\right]$

Proof. The lower bound is easily verified. Since the entries in the sum of Eq. (28) are all non-negative, any finite sum of non-zero entries can be used to obtain a lower bound. The more non-zero entries are used in this finite sum, the sharper this lower bound becomes. The above lower bound is based on a single term of the sum, namely the one that belongs to $z=a$. The expression given for $P\left(\check{a}_{B}=a\right)$ follows by setting $z=a$ in the PMF of theorem 2 .

To prove the upper bound we recall that $P\left(\chi^{2}(p, \lambda) \leq \beta^{2}\right) \leq P\left(\chi^{2}(p, 0) \leq \beta^{2}\right), \forall \lambda>0$. An upper bound is therefore obtained when all $\lambda_{z}$ in Eq. (28) are set to zero. The result then follows by recognizing that all probability masses of the PMF sum up to 1 .

Note that the two bounds relate the probability of the bootstrapped baseline estimator to that of the conditional estimator and to the bootstrapped succes rate. The above bounds become tight when the ambiguity success rate approaches one. This shows, although the probability of the conditional estimator always overestimates the probability of the bootstrapped baseline estimator, that the two probabilities are close for large values of the success rate. This implies that in case of GNSS ambiguity resolution, we should first evaluate the bootstrapped success rate $P\left(\check{a}_{B}=a\right)$ and make sure that its value is close enough to one, before making any inferences on the basis of the distribution of the conditional baseline estimator. In other words, the (unimodal) distribution of the conditional estimator is a good approximation to the (multimodal) distribution of the bootstrapped baseline estimator, when the success rate is sufficiently close to one.

Up this point we have been working with the complete vector $b \in R^{p}$. But recall that vector $b$ contains all real-valued parameters of the GNSS model. It may therefore contain, for instance, apart from the receiver baseline components, also ionospheric or tropospheric delays. In practical applications we may not be interested in the entire vector $b$, but instead in only some of its components, or more generally, in some linear functions of $b$. If that is the case, we would like to have similar results available as given in the above two corollaries, but now specifically applicable to the linear functions we are interested in. That this is possible is shown in the following corollary, the proof which goes along the same lines as the proof of corollary 5 .

Corollary 7 (the linear function bootstrapped probability of concentration). Let $\breve{f}_{B}=F^{T} \breve{b}_{B}$, with $F$ a $p \times q$ matrix of rank $q$, be the ambiguity bootstrapped based estimator of the $q$ linear functions $f=F^{T} b$. Then

$$
P\left(\check{f}_{B} \in T\right)=\sum_{z \in Z^{n}} P\left(\chi^{2}\left(q, \mu_{z}\right) \leq \gamma^{2}\right) P\left(\check{a}_{B}=z\right)
$$

with

$$
\begin{aligned}
T & =\left\{x \in R^{q} \mid\left(x-F^{T} b\right)^{T}\left(F^{T} Q_{\hat{b} \mid \hat{a}} F\right)^{-1}\left(x-F^{T} b\right) \leq \gamma^{2}\right\} \\
\mu_{z} & =\nabla \check{b}_{z}^{T} F\left(F^{T} Q_{\hat{b} \mid \hat{a}} F\right)^{-1} F^{T} \nabla \check{b}_{z}
\end{aligned}
$$

From this result it also follows that, analogous to Eq. (29), similar lower bounds and upper bounds can be formulated for $P\left(\breve{f}_{B} \in T\right)$.

To conclude this section we finally remark, as was mentioned earlier, that the outcome of bootstrapping depends on the chosen ambiguity parametrization. Bootstrapping of DD ambiguities, for instance, will produce an integer solution which generally differs from the integer solution obtained from bootstrapping of reparametrized ambiguities. Since this dependency also holds true for the bootstrapped PMF, we still have some important degrees of freedom left for improving Eqs. (28) and (30) or for sharpening the lower bound of Eq. (29) or its equivalent in the case that linear functions of $b$ are taken.

In order to improve the bootstrapped success rate, we should work with decorrelated ambiguities instead of with the original ambiguities. The method of bootstrapping performs relatively poorly, for instance, when applied to the DD ambiguities. This is due to the usually high correlation between the DD ambiguities. Bootstrapping should therefore be used in combination with the decorrelating $Z$-transformation of the LAMBDA method. This transformation decorrelates the ambiguities further than the best reordering would achieve and thereby reduces the values of the sequential conditional variances. By reducing the values of the sequential conditional variances, the bootstrapped success rate is increased.

For more information on the method, the reader is referred to Teunissen $(1993,1995)$ and de Jonge and Tiberius (1996a) or to the textbooks of HofmannWellenhof et al. (1997), Strang and Borre (1997) and Teunissen and Kleusberg (1998). Practical results obtained with it can be found, for example, in Han (1995), Tiberius and de Jonge (1995), de Jonge and Tiberius (1996b), de Jonge et al. (1996), Boon and Ambrosius (1997), Boon et al. (1997), Tiberius et al. (1997), Jonkman (1998), Cox and Brading (1999) and Peng et al. (1999).

\section{References}

Boon F, Ambrosius B (1997) Results of real-time applications of the LAMBDA method in GPS based aircraft landings. Proc KIS97, pp 339-345

Boon F, de Jonge PJ, Tiberius CCJM (1997) Precise aircraft positioning by fast ambiguity resolution using improved troposphere modelling. Proc ION GPS-97, vol 2, pp 1877-1884

Cox DB, Brading JDW (1999) Integration of LAMBDA ambiguity resolution with Kalman filter for relative navigation of spacecraft. Proc ION NTM 99, pp 739-745

de Jonge PJ, Tiberius CCJM (1996a) The LAMBDA method for integer ambiguity estimation: implementation aspects. LGRSeries no. 12. Delft Computing Centre, Delft 
de Jonge PJ, Tiberius CCJM (1996b) Integer estimation with the LAMBDA method. In: Beutler G. et al. (eds) Proc IAG Symp 115 , GPS trends in terrestrial, airborne and spaceborne applications. Springer, Berlin Heidelberg New York, pp 280-284

de Jonge PJ, Tiberius CCJM, Teunissen PJG (1996) Computational aspects of the LAMBDA method for GPS ambiguity resolution. Proc ION GPS-96, pp 935-944

Fleming W (1977) Functions of several variables, 2nd edn. Springer, Berlin Heidelberg New York

Han S (1995) Ambiguity resolution techniques using integer leastsquares estimation for rapid static or kinematic positioning. Symp Satellite Navigation Technology: 1995 and beyond, Brisbane

Hofmann-Wellenhof B, Lichtenegger H, Collins J (1997) Global positioning system: theory and practice, 4th edn. Springer, Berlin Heidelberg New York

Jonkman NF (1998) Integer ambiguity estimation without the receiver-satellite geometry. LGR-Series no. 18. Delft Geodetic Computing Centre, Delft

Leick A (1995) GPS satellite surveying, 2nd edn. John Wiley, New York

Parkinson B, Spilker JJ (eds) (1996) GPS: theory and applications, vols 1 and 2. AIAA, Washington DC

Peng HM, Chang FR, Wang LS (1999) Attitude determination using GPS carrier phase and compass data. Proc ION NTM 99, pp 727-732

Strang G, Borre K (1997) Linear algebra, geodesy, and GPS. Wellesley-Cambridge Press

Teunissen PJG (1990) GPS op afstand bekeken. In: Snellius Anniversary Volume Een halve eeuw in de goede richting. Delft, pp 215-233

Teunissen PJG (1993) Least-squares estimation of the integer GPS ambiguities. Invited Lecture, Sect IV Theory and Methodology, IAG General Meeting, Beijing, August. Also in: LGR Series no. 6. Delft Geodetic Computing Centre, Delft

Teunissen PJG (1995) The least-squares ambiguity decorrelation adjustment: a method for fast GPS integer ambiguity estimation. J Geod 70: 65-82

Teunissen PJG (1998) On the integer normal distribution of the GPS ambiguities. Artific Sat 33(2): 49-64

Teunissen PJG (1999) The probability distribution of the GPS baseline for a class of integer ambiguity estimators. J Geod 73: 275-284

Teunissen PJG, Kleusberg A (eds) (1998) GPS for geodesy, 2nd enlarged edn. Springer, Berlin Heidelberg New York

Tiberius CCJM, de Jonge PJ (1995) Fast positioning using the LAMBDA method. Proc DSNS-95, paper 30

Tiberius CCJM, Teunissen PJG, de Jonge PJ (1997) Kinematic GPS: performance and quality control. Proc KIS97, pp 289-299 